\title{
Adaptive optics upgrades for laser communications to the ESA optical ground station
}

E. Fischer, K. Kudielka, T. Berkefeld, D. Soltau, J. Perdigués-Armengol, et al.

E. Fischer, K. Kudielka, T. Berkefeld, D. Soltau, J. Perdigués-Armengol, Z. Sodnik, "Adaptive optics upgrades for laser communications to the ESA optical ground station," Proc. SPIE 11852, International Conference on Space Optics - ICSO 2020, 118522A (11 June 2021); doi: 10.1117/12.2599370

SPIE Event: International Conference on Space Optics - ICSO 2021, 2021, Online Only 


\section{International Conference on Space Optics-ICSO 2020}

Virtual Conference

30 March-2 April 2021

Edited by Bruno Cugny, Zoran Sodnik, and Nikos Karafolas
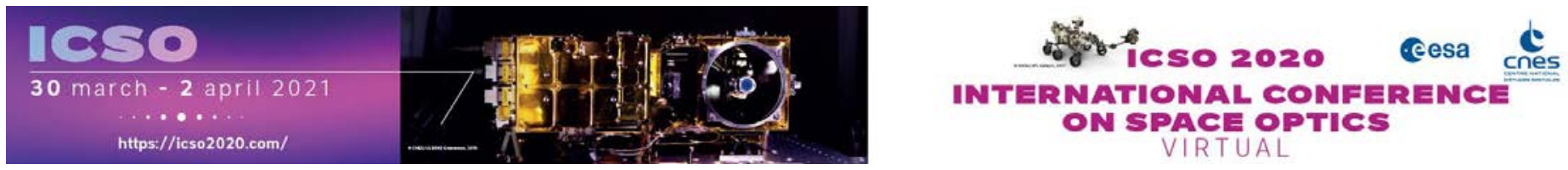

\section{Adaptive optics upgrades for laser communications to the ESA optical ground station}

\section{Cesa isoporecestings denes}




\title{
Adaptive Optics Upgrades for Laser Communications to the ESA Optical Ground Station
}

\author{
E. Fischer ${ }^{* a}$, K. Kudielka ${ }^{\text {a }}$, T. Berkefeld ${ }^{\mathrm{a}}$, D. Soltau ${ }^{\mathrm{a}}$, J. Perdigues-Armengol ${ }^{\mathrm{b}}$, Z. Sodnik $^{\mathrm{b}}$ \\ aSynopta GmbH, Sonderstrasse 7, 9034 Eggersriet, Switzerland; \\ ${ }^{b}$ ESA/ESTEC, European Space Agency Noordwijk, The Netherlands
}

\begin{abstract}
Over the past years, Synopta have successfully applied adaptive optics (AO) in optical ground stations to enhance the capabilities in satellite-to-ground laser communication links. In this paper, we present the outcome of an upgrade activity to the ESA Optical Ground Station (ESA OGS). The activities comprise the design, fabrication and site acceptance test results of the Cassegrain Adaptive Receiver Optics ("CARO") add-on instrument to the $1 \mathrm{~m}$ telescope of the ESA OGS. Additionally, we present a baseline design concept for the future use of a bi-directional adaptive optics system in the Coudé path of the ESA OGS, supporting pre-distortion of the uplink beam, with expected performance characteristics for a selected uplink application.
\end{abstract}

Keywords: adaptive optics, deformable mirror, optical ground station, wavefront sensor

\section{INTRODUCTION}

ESA has realized in the frame of the SILEX program a stationary optical ground station (OGS). It is located at the Observatorio del Teide, Izaña, Tenerife (Spain), at an altitude of $2391 \mathrm{~m}$ above sea level. The station was initially designed for in-orbit check-out and testing of the laser communication payload embarked on the ARTEMIS spacecraft in geostationary orbit. This OGS is equipped with an English mounted $1 \mathrm{~m}$ Zeiss telescope. The telescope can be operated in either a Ritchey-Chretien or a Coudé configuration.

Consequently, a second generation of Laser Communication Terminals (LCT) with improved mass and data rate figures has been developed by Tesat Spacecom under contract of the German Space Agency (DLR). In the frame of the European Data Relay Satellite (EDRS) system development the second generation LCT has been adopted also for interorbit applications, i.e. LEO to GEO free space optical (FSO) communication [2]. In order to exploit the full capacity potential of satellite based FSO communication systems it is further necessary to establish also optical satellite-to-ground links (SGL) and vice versa.

In the past the ESA OGS could only be used to support the in-orbit check-out of coherent optical FSO communication activities. Its $1 \mathrm{~m}$ receive telescope diameter is significantly larger than the expected Fried parameter, i.e. $\mathrm{r}_{0}$. In order to overcome this limitation an upgrade activity has been initiated so that the ESA OGS can in the future establish also coherent optical FSO communication links with GEO as well as with LEO satellites in the $1064 \mathrm{~nm}$ or $1550 \mathrm{~nm}$ band, respectively.

The chosen approach to make the $1 \mathrm{~m}$ aperture of the ESA OGS usable for reception of coherent laser light was the implementation of an adaptive optics system (AO). The AO implementation builds on the heritage and field experience gained with TAOGS, a transportable optical ground station equipped with an AO system, that has been developed and manufactured by Synopta under Tesat contract for DLR. [4]. In receive direction of the TAOGS, an adaptive optical system is implemented to correct the received distorted wavefront.

The design and factory acceptance testing of CARO has been described in detail elsewhere [6]. In $\$ 2$ we focus on commissioning results and provide only a short CARO design summary. In $\$ 3$ we describe a future bi-directional adaptive optics system design for the Coudé path of the ESA OGS.

*edgar.fischer@synopta.ch; www.synopta.ch 


\section{CASSEGRAIN ADAPTIVE RECEIVER SYSTEM (CARO)}

\subsection{Design Summary}

The CARO instrument is an adaptive optics system for stationary usage which shall correct in the ESA-OGS telescope the received optical wavefront such that it can be coupled into a single mode optical fiber. Therefore, the CARO system is designed to pick-up at its entrance port from the OGS telescope a blurred primary Cassegrain focus spot. The received light is delivered at the CARO exit port by a polarization-maintaining single mode fiber. The CARO system is designed for operation in two spectral bands:

- $\quad$ CARO spectral band $\mathrm{I}$ is ranging from $1030 \mathrm{~nm}$ to $1080 \mathrm{~nm}$

- $\quad$ CARO spectral band II is ranging from $1530 \mathrm{~nm}$ to $1570 \mathrm{~nm}$

The system design is made such that CARO achieves a Strehl ratio of 0.6 at a $\mathrm{r}_{0}$-value of $5 \mathrm{~cm}(\lambda=1064 \mathrm{~nm})$. The maximum specified operational ground wind speed is $50 \mathrm{~km} / \mathrm{h}$.

The CARO system is designed to be embedded into the overall OGS system during SGLs. Figure 1 shows schematically how CARO is embedded into the ESA OGS system.

The CARO software is implemented as server/client application. The user can operate the system from the control room, or even via the Internet, if desired.

The physical realization of CARO (photograph see Figure 2) comprises all the CARO-elements from Figure 1 except the control panel. The control panel software is located in the ESA OGS control computer system. The physical CARO unit can be attached to the backend of the OGS telescope tube at the telescope flange plate.

A collimator picks-up the telescope's primary Cassegrain focus and collimates the received optical beam. A beam splitter is placed behind the collimator. This beam splitter passes a small fraction of the incident light in receive direction (RX) towards the acquisition sensor (AS). The AS is an InGaAs based array sensor. The purpose of the AS is threefold:

- To enable acquisition and centering of the spot caused by the remote communication partner during the spatial acquisition phase

- To keep during the tracking phase the spot of the remote communication partner centered in the field of view of the wavefront sensor, via OGS telescope off-set commanding.

- To support the acquisition of a telescope pointing model by means of star imaging.

The Rx light reflected at the aforementioned beamsplitter propagates further towards the fine pointing assembly (FPA). The FPA is used for line-of-sight stabilization. A relay optics transfers the telescope pupil located onto the FPA mirror to the deformable mirror's (DM) active mirror surface. The DM is equipped with 24 actuators across the diameter. The total actuator count of the DM amounts to 468. A second afocal relay optics is finally used to relay the pupil from the DM to the lenslet array of a Shack-Hartmann wavefront sensor (WFS).

The light required by the WFS is picked-off from the RX light which travels towards the Rx-fiber. In Figure 3 the hardware of the CARO optical bench is depicted. The photograph has been taken prior to box closure. 


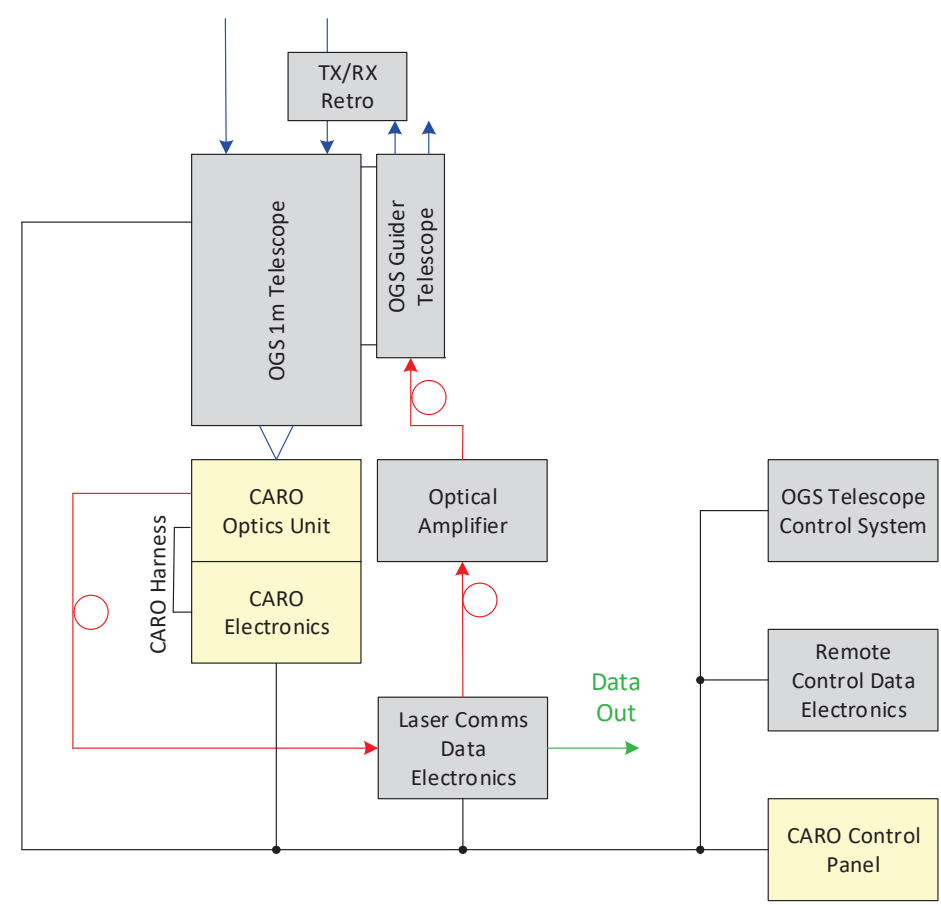

Figure 1. Block diagram depicts how CARO is embedded into ESA OGS when configured for satellite-to-ground links.

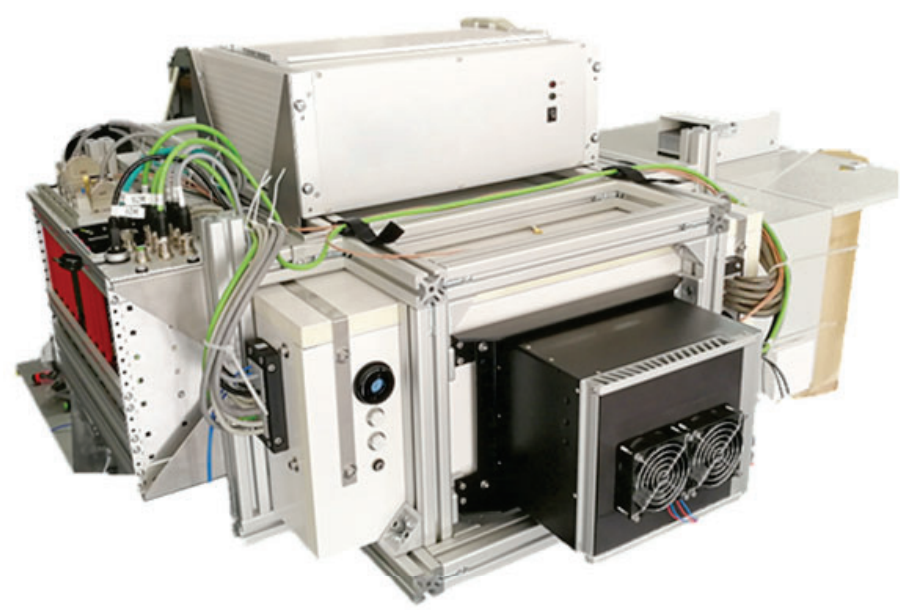

Figure 2. CARO instrument (Optical Bench and calibration laser sources are inside the Housing). 


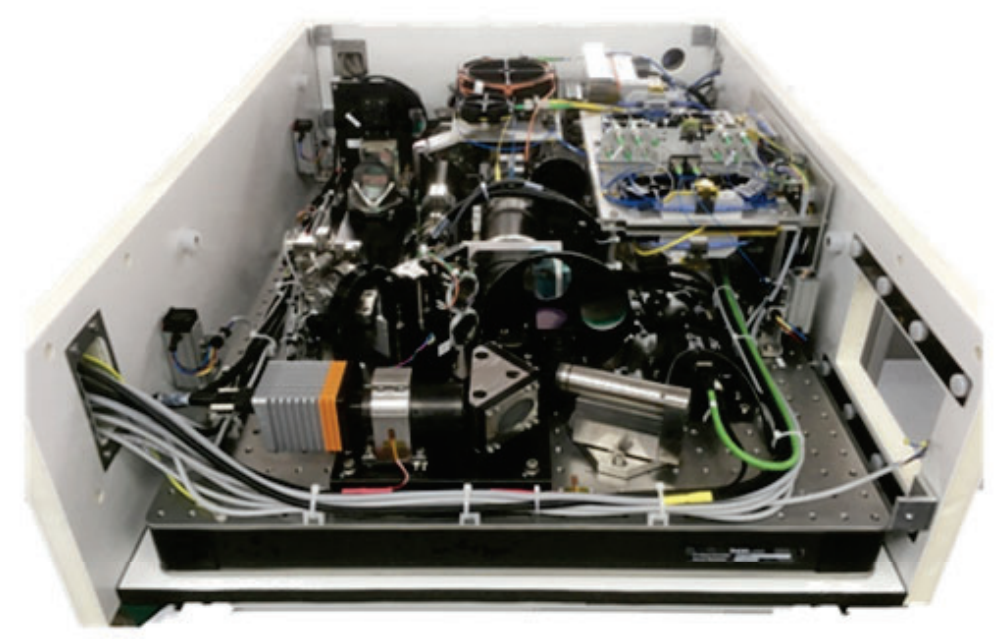

Figure 3. CARO instrument optical bench before box closure.

\subsection{Factory Acceptance Tests}

Prior to the shipment of CARO to the ESA OGS a factory acceptance test (FAT) demonstrated the full functionality of the instrument. During the FAT a telescope simulator in combination with a rotating phase plate was used. The telescope simulator had the same f-number than the $1 \mathrm{~m}$ telescope at ESA OGS. The rotating phase plate was used to simulate the turbulence caused by the boundary layer. By varying the rotational speed of the phase plate different cross-wind speeds could be simulated.

The measurement CARO insertion loss, i.e. transmission without turbulence, showed values between 0.34 and 0.388 . This means that the CARO system achieves almost the theoretical limit of 0.39 (limit derived from optical transmission budget and fiber coupling efficiency in the presence of a centrally obscured pupil) in terms of fiber coupling efficiency.

In a second test sequence the corrected beam quality in terms of average Strehl ratio was measured with simulated turbulence. The achieved Strehl ratio varied between 0.4 (max. wind speed at $\lambda=1060 \mathrm{~nm}$ ) and 0.83 (typical wind speed at $\lambda=1550 \mathrm{~nm})$.

\subsection{Commissioning at ESA OGS}

The CARO instrument was shipped in autumn 2019 to the ESA OGS and installed to the $1 \mathrm{~m}$ telescope. The instrument commissioning at the ESA OGS took place in November 2019 (see Figure 4). After the installation and commissioning of CARO several test links with the technology demonstration package \#1 (TDP\#1) on board the Alphasat satellite in Geostationary Earth Orbit (GEO, location is 25 degrees East) have been performed. 


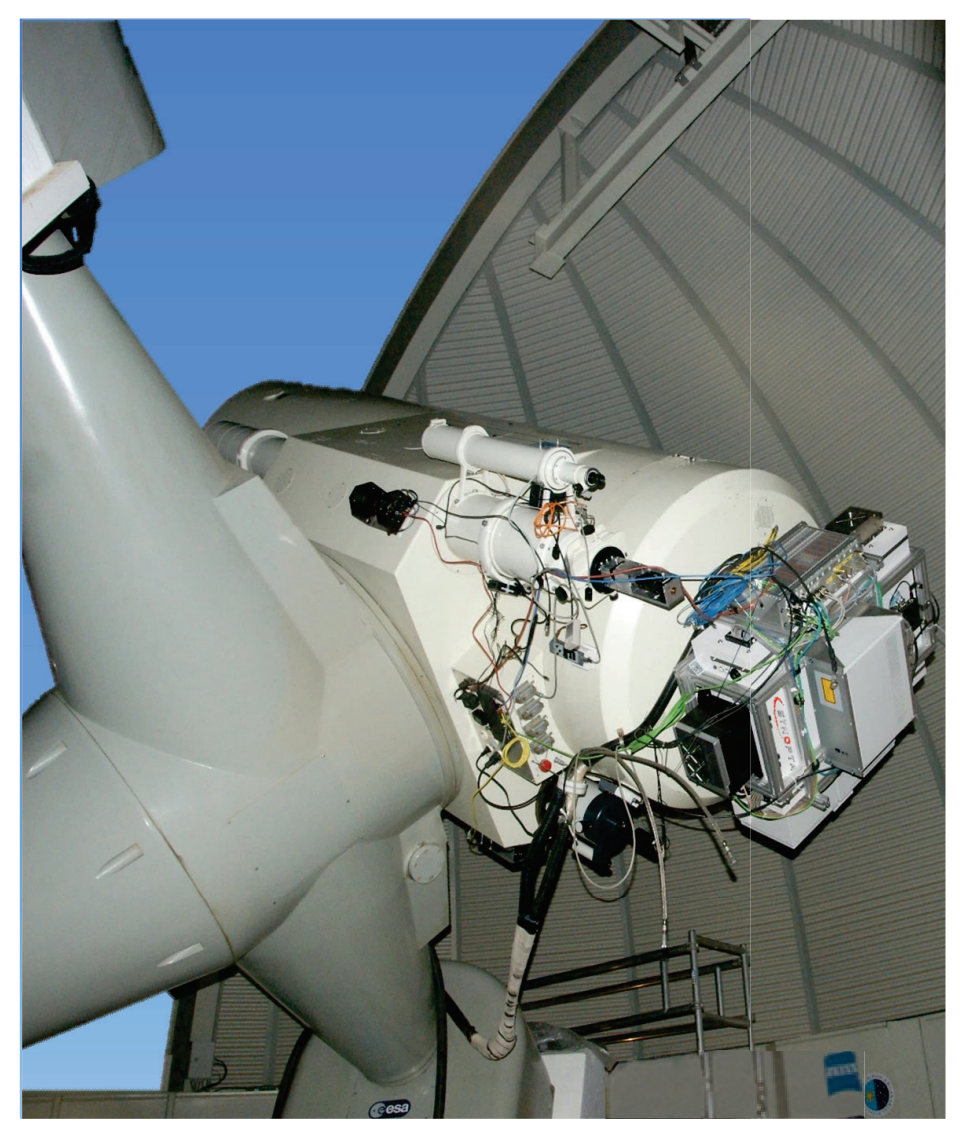

Figure 4. CARO instrument mounted to ESA OGS telescope.

During the spatial acquisition phase, the automated acquisition and centering of the Alphasat terminal in the FoV of CARO worked as expected. During the test links it was demonstrated that sufficient optical power is available in the CARO output fibre and even error free communication in downlink as well as in uplink direction was achieved. In Figure 5 a screenshot of the main user interface is shown when running the AO. CARO is equipped with a scoring camera, called JCAM. The JCAM is located in an optical path parallel to the wavefront sensor. In Figure 6 a screen shot of the scoring camera is depicted which was taken during the link tests. A nearly diffraction limited spot can be seen.

With the test links performed to/with Alphasat LCT during the commissioning phase the functional performance of CARO could be demonstrated. 


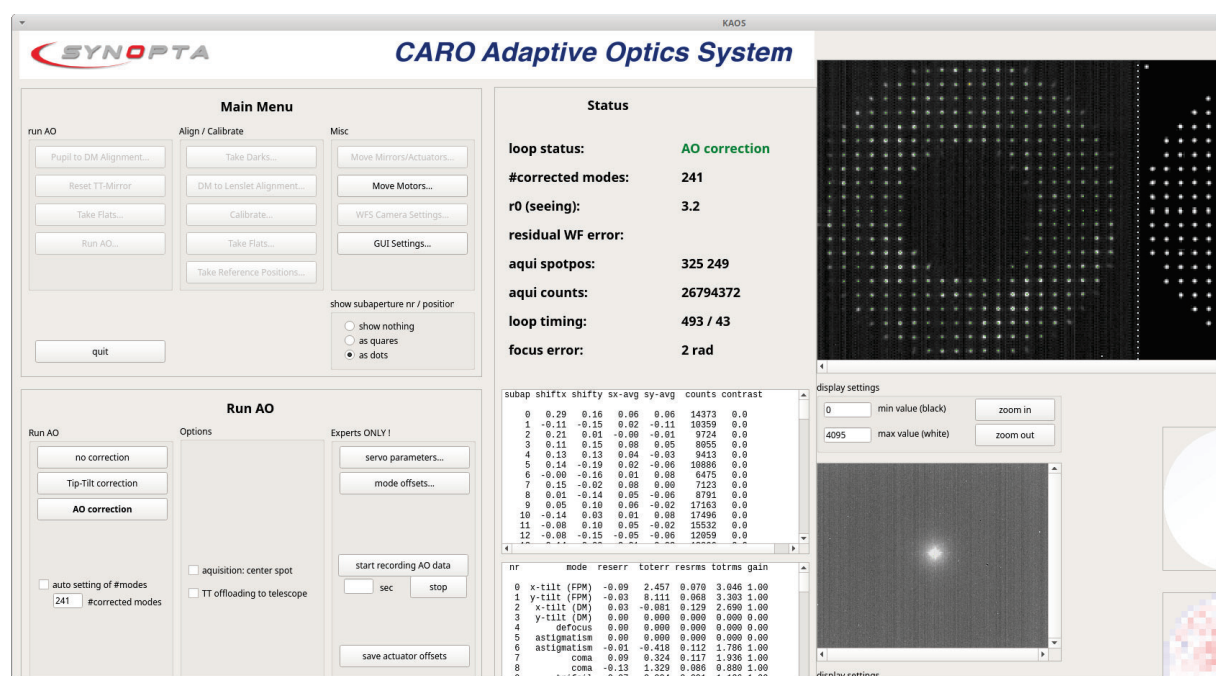

Figure 5. Main user interface, with "Run AO” menu.

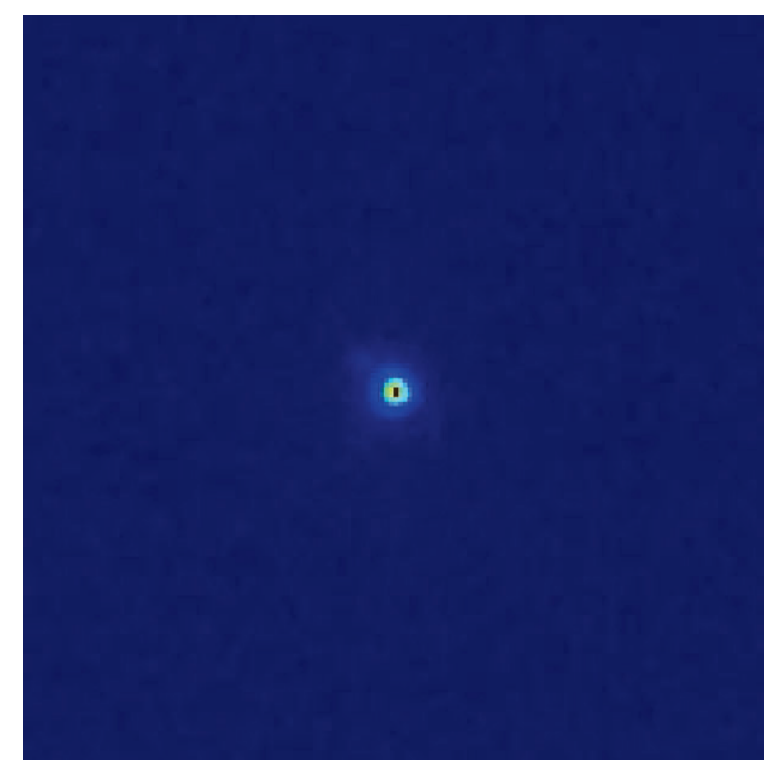

Figure 6. AlphaSat TDP-1 LCT observed with CARO JCAM and AO active

\subsection{Acceptance Testing}

The acceptance test campaign was originally scheduled for spring 2020 . However, due to Covid-19 induced restrictions this campaign could not be performed as planned in 2020. The campaign had therefore to be postponed to April 2021. 


\section{FUTURE BI-DIRECTIONAL ADAPTIVE OPTICS SYSTEM DESIGN FOR THE COUDÉ PATH OF THE ESA OGS}

Part of the ESA OGS upgrade activity was also to design a future Coudé Laser Communication System (CLCS) for optical feeder links. The preliminary design of such a system has been described in [6]. The final design has been modified w.r.t. the preliminary design in the following points:

- Now the full OGS telescope aperture is used in receive direction

- In transmit direction in each quadrant of the OGS telescope aperture a transmit beam with $20 \mathrm{~cm}$ beam diameter is transmitted (see Figure 7). Each of these TX beams covers a different wavelength range, and also carries different data.

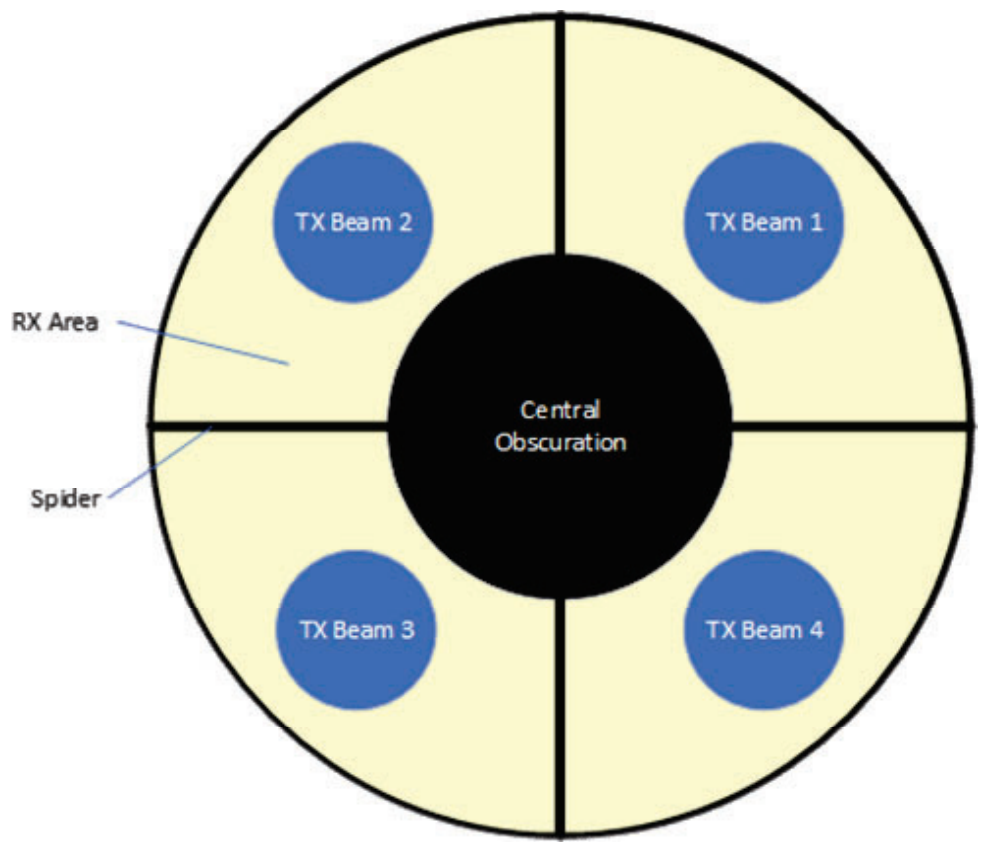

Figure 7. Scaled representation of the size ratios in the OGS telescope aperture

It is well known from literature, that tip-tilt only corrected uplinks will suffer from significant scintillations, if the ratio of aperture diameter and Fried parameter $(\mathrm{D} / \mathrm{r} 0)$ is much larger than 1. Furthermore, we have shown by analysis that, with full pre-compensation, relatively benign scintillation can be achieved with higher $\mathrm{D} / \mathrm{r} 0$ ratios (even with the required GEO point-ahead, [7]. So, at a given seeing, we are able to increase transmit antenna gain, when using full precompensation. Instead of the originally selected DM with 69 actuators in the revised design a deformable mirror with a significantly increased actuator count is required, e.g. a DM468 from ALPAO.

When using full pre-compensation in a feeder uplink to Alphasat TDP-1, it should be possible to exceed the minimum required power in the LCT's input aperture $(-44 \mathrm{dBm})$ at high confidence, with margin, at a booster amplifier output power of only $2.5 \mathrm{~W}$.

The revised top level CLCS optical block diagram is shown in Figure 8. The system comprises the following optical paths: 
- $\quad$ Receive direction

- Acquisition sensor path, used for initial large field of view spatial acquisition

- Fine acquisition sensor path, used for fast re-acquisition and/or tip/tilt tracking

○ Wavefront sensing path, used for AO wavefront sensing

- RX fiber path, used for collecting received communication light in a single-mode fiber

- Transmit direction

- TX beam path, used for transmitting four spatially separated TX sub-beams in the common TX/RX path section

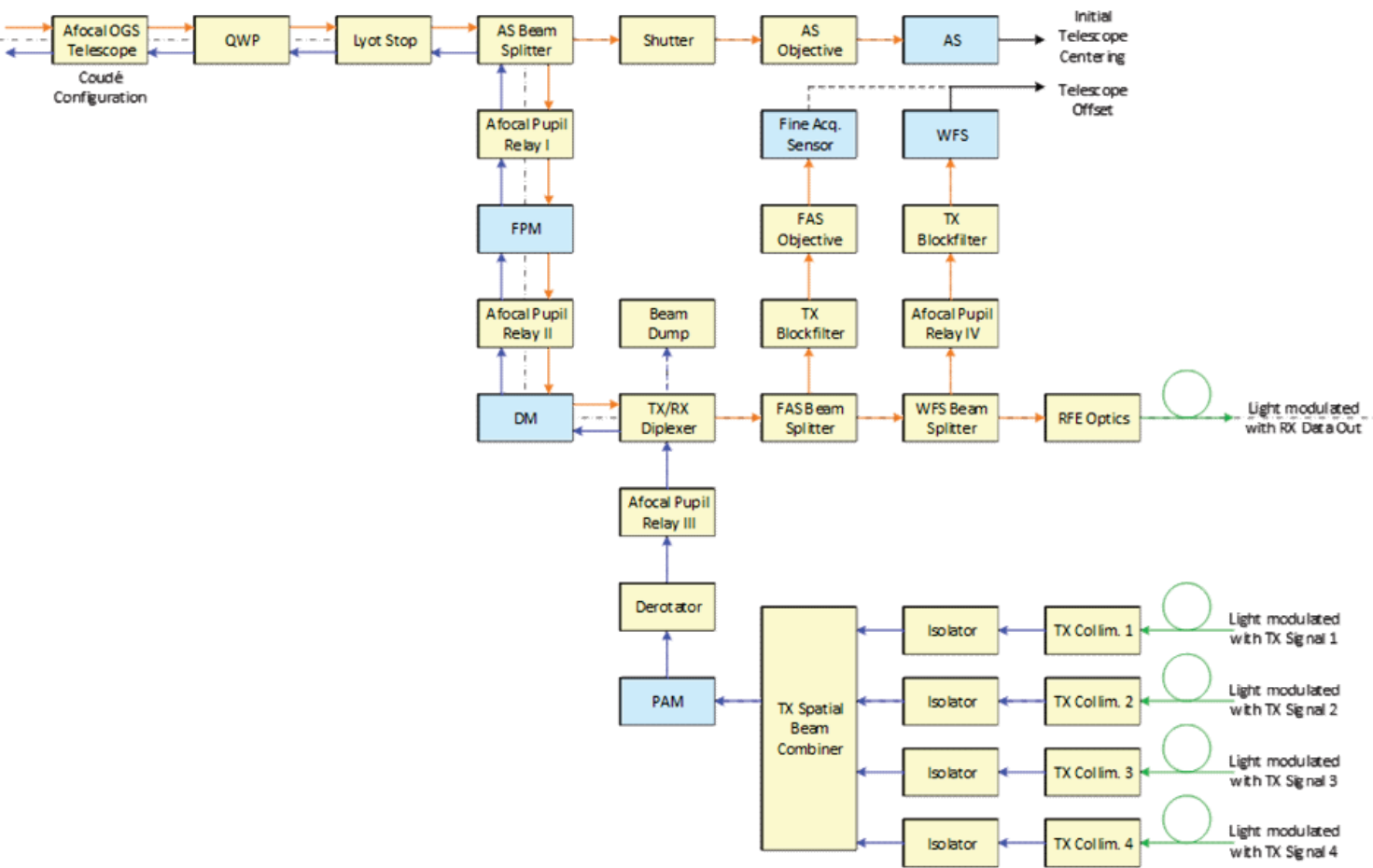

Figure 8. Revised CLCS optical block diagram

The optical layout of the RX fiber path on the Coudé bench starts at the telescope's primary focus. As beam collimator a slightly tilted spherical mirror is used. A Lyot stop has been added in the optical path to allow a realistic factory acceptance testing of the optics before shipment to the OGS. The size of the Lyot stop and the pupil size at the DM have been chosen to be identical. Therefore, two identical afocal relay telescopes can be used as pupil shifters. The first of these relay telescopes is imaging the Lyot stop onto the FPM. This imaging process is performed with a magnification of less than unity. Therefore, the second telescope must be implemented with a reversed order concerning the mirror arrangement. Because the second telescope images the pupil from the FPM to the DM and during this second imaging process the pupil size is magnified back to the original size than at it was the Lyot stop.

For the afocal relay telescope design preference has been given to a design that comprises two off-axis mirrors rather than to a design that comprises two achromats with in total 8 optical surfaces. The relay telescopes are implemented as afocal off-axis Gregorian type telescopes. The fiber coupling lens is a custom doublet design derived from the CARO fiber coupling lens. The system is very well corrected over the whole optical C-band. 
The optical layout of the TX path on the Coude bench is woven into the RX path described above. A front surface polarizing beam splitter (PBS) plate with a high extinction ratio is used as TX/RX beam splitter. An optical beam dump is placed behind the unused TX/RX beam splitter port to stop leaking TX light from further propagation into the system.

A refractive afocal pupil relay telescope is used to relay the pupil from the DM onto the point ahead mirror (PAM). The optics will be used a) only for TX light and b) practically only on-axis. Therefore, one can use in this case a refractive solution. Two commercial achromats are used for this purpose.

An image derotator mechanism is required to ensure that the TX beams are staying stationary w.r.t. to the telescope spider structure for any declination and hour angle position of the telescope. The derotator consists of either three flat mirrors or a Dove prism. The compactly folded optical layout of the CLCS breadboard does not allow to place the derotator in the common TX/RX path section.

\section{CONCLUSIONS}

In this paper we've presented the successful installation and commissioning of CARO at the $1 \mathrm{~m}$ telescope of the ESA OGS on Tenerife. In the second half of this paper the design of a future Coudé Laser Communication System for optical feeder links is described that resides on the Coudé bench of the ESA OGS.

\section{REFERENCES}

[1] Tolker-Nielsen T., Oppenhauser G., "In-orbit test result of an operational optical inter satellite link between ARTEMIS and SPOT4, SILEX”., Proc. SPIE, 2002, vol. 4635, pp.1-15.).

[2] Tröndle D. et al., "Optical LEO-GEO Data Relays: From Demonstrator to Commercial Application”, 32nd AIAA International Communications Satellite Systems Conference, AIAA SPACE Forum, (AIAA 2014-4439).

[3] Gregory M. et al., "TESAT Laser Communication Terminal Performance Results on 5.6 GBIT Coherent Inter Satellite and Satellite to Ground Links", ICSO 2010 International Conference on Space Optics, Rhodes, Greece, 4 - 8 October 2010

[4] Fischer E. et al., "Development, Integration and Test of a Transportable Adaptive Optical Ground Station", 2015 IEEE International Conference on Space Optical Systems and Applications (ICSOS), DOI: 10.1109/ICSOS.2015.7425071

[5] Saucke K. et al., "The Tesat Transportable Adaptive Optical Ground Station", Proc. SPIE 9739, Free-Space Laser Communication and Atmospheric Propagation XXVIII, 973906 (15 March 2016).

[6] Fischer E. et al., "ESA Optical Ground Station Upgrade with Adaptive Optics for High Data Rate Satellite-toGround Links -Test Results", 2019 IEEE International Conference on Space Optical Systems and Applications (ICSOS), DOI: 10.1109/ICSOS45490.2019.8978974

[7] K. Kudielka et al.; Numerical prediction and experimental validation of irradiance fluctuations in a precompensated optical feeder link, Poster, Proc. ICSO 2018. 